\title{
Maspin expression is frequent and correlates with basal markers in triple-negative breast cancer
}

\author{
Yoshihisa Umekita1*, Yasuyo Ohi², Masakazu Souda', Yoshiaki Rai ${ }^{2}$, Yoshiaki Sagara², Yasuaki Sagara², \\ Shugo Tamada ${ }^{2}$ and Akihide Tanimoto ${ }^{1}$
}

\begin{abstract}
Background: Maspin is a unique member of the serine protease inhibitor superfamily and its expression is found in myoepithelial cells of normal mammary glands; therefore, it has been considered to be a myoepithelial marker. We previously reported that maspin was frequently expressed in biologically aggressive breast cancers. In turn, triple-negative (TN) breast cancer is a subtype of tumor with aggressive clinical behavior and shows frequent expression of basal markers. We hypothesized that maspin expression may be frequent and correlate with basal rather than myoepithelial markers in TN breast cancer.
\end{abstract}

Methods: Paraffin-embedded 135 TN invasive ductal carcinoma tissue samples were immunohistochemically investigated using the Dako Envision+ kit and primary antibodies for maspin, basal (CK5/6, EGFR, CK14) and myoepithelial markers (p63, CD10). The correlation between maspin expression and relapse-free survival (RFS) was investigated by the log-rank test.

Results: The positive rate for maspin was $85.9 \%$ and significantly correlated with younger age $(P=0.0015)$, higher histological grade $(P=0.0013)$, CK5/6 positivity $(P<0.0001)$, CK14 positivity $(P=0.0034)$ and the basal-like subtype defined by CK5/6, EGFR and CK14 positivity $(P=0.013)$. The positive rates for CK5/6, EGFR, CK14, CD10 and p63 were $59.2 \%, 48.9 \%, 34.1 \%, 17.8 \%$ and $12.6 \%$, respectively. There was no significant correlation between maspin expression and RFS.

Conclusions: The positive rate for maspin is the highest among known basal and myoepithelial markers, and strongly correlates with basal markers in TN breast cancer. These results suggested that maspin could be a candidate for a therapeutic target for TN breast cancer.

\section{Introduction}

Maspin is a unique member of the serine protease inhibitor superfamily and it has been shown to have tumor suppressive activity attributable to the inhibition of breast cancer cell motility, invasion and metastasis [1]. Its expression is found in myoepithelial cells of normal mammary glands; therefore, it has been considered to be a myoepithelial marker, but its correlation with basal markers, such as CK5/6, CK14 and epidermal growth factor receptor (EGFR), in breast cancers remains to be solved. On the other hand, triple-negative (TN) breast cancer is a subtype of tumor with aggressive clinical

\footnotetext{
* Correspondence: umekita@m2.kufm.kagoshima-u.ac.jp

'Department of Molecular and Cellular Pathology, Field of Oncology, Kagoshima University Graduate School of Medical and Dental Sciences 8-351, Sakuragaoka, Kagoshima 890-8544, Japan

Full list of author information is available at the end of the article
}

behavior which currently lacks effective targeted therapies [2]; however, TN breast cancer encompasses a remarkably heterogeneous group of tumors, and the expression of basal markers identifies biologically and clinically distinctive subgroups of TN tumors [2]. We previously reported that maspin expression was an independent poor prognostic indicator in invasive ductal carcinoma (IDC) [3], and that its expression was upregulated during the progression of mammary ductal carcinoma [4]. Additionally, Rakha et al. reported that basal, not myoepithelial, phenotypes defined by CK5/6 and/or CK14 positivity had an independent value in predicting a poor clinical outcome in a large number of invasive breast carcinomas [5]. Taken together, we hypothesized that maspin expression could be frequent and correlated with basal rather than myoepithelial markers in TN breast cancer. To explore this hypothesis, we 
investigated the frequency of maspin expression and its correlation with established basal (CK5/6, EGFR, CK14) and myoepithelial (p63, CD10) markers in TN breast cancer. In addition, we investigated the relationship between maspin expression and relapse-free survival (RFS) in TN breast cancer.

\section{Materials and methods}

Paraffin-embedded tissue samples obtained from 135 TN breast cancer patients between Descember 2001 and March 2006 were collected from Hakuaikai Sagara Hospital (Kagoshima, Japan). All breast cancers were histologically classified as IDC. The median age was 56.6 years (range: 27-91 years). Of 135 patients, follow-up data were obtained from 126 patients. The median follow-up time was 64.2 months (range: 3-136 months). Breast cancer recurred in 27 patients (21.4\%) during the follow-up period. All patients, except for one, were histologically examined for axillary lymph node involvement, and 52 patients were histologically diagnosed as node-positive. TN was defined as negative for ER and PgR (cutoff 10\%), as well as HER2 negative (Hercep test: score $0,1+, 2+)$. In cases that scored $2+$, the absence of HER2 gene amplification was confirmed by fluorescent in situ hybridization analysis using the PathVysion kit (Abbott-Vysis, Inc., Downers Grove, IL). Immunohistochemistry was performed using the Dako Envision+ kit in conjunction with the DAKO Autostainer according to the instructions supplied by the manufacturer, as described previously [6]. The primary antibodies used and their cutoff values are shown in Table 1 . The basallike subtype was defined by positive for CK5/6 and/or EGFR [7]. Each staining result was assessed independently by two pathologists (YO and YU). When the evaluations differed, final agreement was reached by consensus. The patients and their tumor characteristics were analyzed using the chi-square test. Actuarial curves for RFS were calculated by the Kaplan-Meier technique. RFS were calculated from the date of first surgery to the date of clinical or pathological relapse. Differences in RFS were tested with the log-rank test. All statistical analyses were performed with a statistical software package (Dr SPSS version 11.0.1J; SPSS Japan Inc., Tokyo, Japan). The cutoff for significance was taken as $P=0.05$.

\section{Results}

Patient and tumor characteristics are summarized in Table 2. The positive rates for maspin, EGFR, CK5/6, CK14, CD10 and p63 were 85.9\% (116 cases), 48.9\% (66 cases), $59.3 \%$ (80 cases), 34.1\% (46 cases), 17.8\% (24 cases) and $12.6 \%$ (17 cases), respectively (Table 2 ). Expression of maspin significantly correlated with younger age $(P=0.0015)$, higher histological grade $(P=$ $0.0013)$, CK5/6 positivity $(P<0.0001)$ and CK14 positivity $(P=0.0034)$ (Table 3$)$. The representative positive staining pattern of maspin is shown in Figure 1. The myoepithelial cells and stromal cells in mammary glands served as internal positive and negative controls, respectively. The basal-like subtype defined by CK5/6 and/or EGFR expression was observed in $76.3 \%$ (103 of 135 cases). The positivity for maspin showed a slightly significant correlation with the basal-like subtype $(P=$ 0.041) (Table 4). When defined by CK5/6 and/or EGFR and/or CK14 positivity, the basal-like subtype was found in $79.3 \%$ (107 of 135 cases) and the positivity for maspin more significantly correlated with the basal-like subtype $(P=0.013)$ (Table 4$)$. The log-rank test showed that only node metastases significantly correlated with RFS $(P<0.0001)$. There was no significant correlation between maspin expression and RFS $(P=0.204)$.

\section{Discussion}

In the current study, we restricted our analysis to IDC of no special type to avoid any confounding effect of special types of invasive breast cancer, such as lobular, medullary and metaplastic carcinomas. Previous DNA microarray and immunohistochemical analyses showed that $80 \%$ to $90 \%$ of TN breast cancer were basal-like subtypes and had clinical behavior similar to basal-like behavior [2]. The positive rate for maspin in our study was $85.9 \%$ of TN breast cancer, and the positive rate was similar to that of basal-like subtypes in TN breast

Table 1 Souce, dilution, pretreatment and cutoff values of primary antibodies used

\begin{tabular}{|c|c|c|c|c|}
\hline Antibody (clone) & Manufacturer & Dilution & Pretreatment & Cutoff values \\
\hline Maspin (EAW24) & Novocastra & $1: 200$ & Microwave & $\geqq 10 \%$ (positive) \\
\hline CK5/6 (D5/16B4) & Dako & $1: 50$ & Water bath & $\geqq 10 \%$ (positive) \\
\hline CK14 (LL002) & Novocastra & $1: 100$ & Water bath & $\geqq 10 \%$ (positive) \\
\hline EGFR (EGFR.113) & Novocastra & $1: 25$ & Microwave & $\geqq 10 \%$ (positive) \\
\hline CD10 (56C6) & Novocastra & $1: 50$ & Water bath & $\geqq 10 \%$ (positive) \\
\hline p63 (4A4+Y4A3) & LabVision & $1: 200$ & None & $\geqq 10 \%$ (positive) \\
\hline ER (1D5) & Dako & $1: 50$ & Water bath & $\geqq 10 \%$ (positive) \\
\hline PgR (PgR636) & Dako & $1: 800$ & Water bath & $\geqq 10 \%$ (positive) \\
\hline HER2 & Dako & Prediluted (Hercep test) & Water bath & Score $3+$ (positive) \\
\hline
\end{tabular}


Table 2 Patients and tumor characteristics in triplenegative breast cancer cohort

\begin{tabular}{|c|c|c|}
\hline Parameters & Number & Percentage \\
\hline \multicolumn{3}{|l|}{ Age (years) } \\
\hline$\leq 50$ & 51 & 37.8 \\
\hline$>50$ & 84 & 62.2 \\
\hline \multicolumn{3}{|c|}{ Tumor size (mm) } \\
\hline$\leq 20$ & 44 & 32.6 \\
\hline$>20$ & 91 & 67.4 \\
\hline \multicolumn{3}{|c|}{ Lymph node metastases } \\
\hline 0 & 82 & 60.7 \\
\hline $1-3$ & 28 & 20.7 \\
\hline $3<$ & 24 & 17.8 \\
\hline \multicolumn{3}{|c|}{ Histological grade } \\
\hline । & 5 & 3.7 \\
\hline$\|$ & 29 & 21.5 \\
\hline III & 101 & 74.8 \\
\hline \multicolumn{3}{|l|}{ Maspin } \\
\hline Positive & 116 & 85.9 \\
\hline Negative & 19 & 14.1 \\
\hline \multicolumn{3}{|l|}{ EGFR } \\
\hline Positive & 66 & 48.9 \\
\hline Negative & 69 & 51.1 \\
\hline \multicolumn{3}{|l|}{ CK5/6 } \\
\hline Positive & 80 & 59.3 \\
\hline Negative & 55 & 40.7 \\
\hline \multicolumn{3}{|l|}{ CK14 } \\
\hline Positive & 46 & 34.1 \\
\hline Negative & 89 & 65.9 \\
\hline \multicolumn{3}{|l|}{ CD10 } \\
\hline Positive & 24 & 17.8 \\
\hline Negative & 111 & 82.2 \\
\hline \multicolumn{3}{|l|}{ p63 } \\
\hline Positive & 17 & 12.6 \\
\hline Negative & 118 & 87.4 \\
\hline
\end{tabular}

cancer. Although there is no consensus about the definition and method of identification of a basal-like subtype in routine practice, tumors with a basal-like phenotype were strongly associated with a high histological grade in all classifications [2], which is similar to our previous and present findings that maspin positivity strongly correlated with a high histological grade in IDC [3] and TN breast cancer. There is no international consensus on the precise complement of markers that defines a basallike subtype [2], but Nielsen's definition, used in our study, is currently considered one of the most pragmatic and widely accepted definitions of basal-like breast cancer [7]. The single use of maspin positivity was able to detect 89.3\% (92 of 103) of basal-like subtypes defined by Nielsen's definition (CK5/6 and/or EGFR positivity). Including CK14 in Nielsen's definition, 89.7\% (96 of 107) of the basal-like subtypes was detected by the
Table 3 Association between maspin expression and clinicopathological factors in triple-negative breast cancer

\begin{tabular}{|c|c|c|c|}
\hline & Maspin & pression & \\
\hline & $\begin{array}{c}\text { Positive }(85.9 \%) \\
116 \text { cases }\end{array}$ & $\begin{array}{c}\text { Negative }(14.1 \%) \\
19 \text { cases }\end{array}$ & $\begin{array}{c}P- \\
\text { value }\end{array}$ \\
\hline Age (years) & & & \\
\hline$\leq 50$ & 50 & 1 & 0.0015 \\
\hline$>50$ & 66 & 18 & \\
\hline Tumor size (mr & & & \\
\hline$\leq 20$ & 41 & 3 & 0.091 \\
\hline$>20$ & 75 & 16 & \\
\hline $\begin{array}{l}\text { Lymph node } \\
\text { metastases }\end{array}$ & & & \\
\hline 0 & 69 & 13 & 0.226 \\
\hline $1-3$ & 27 & 1 & \\
\hline $3<$ & 20 & 4 & \\
\hline $\begin{array}{l}\text { Histological } \\
\text { grade }\end{array}$ & & & \\
\hline 1 & 4 & 1 & 0.0013 \\
\hline$\|$ & 19 & 10 & \\
\hline III & 93 & 8 & \\
\hline EGFR & & & \\
\hline Positive & 57 & 9 & 0.886 \\
\hline Negative & 59 & 10 & \\
\hline CK5/6 & & & \\
\hline Positive & 77 & 3 & $<0.0001$ \\
\hline Negative & 39 & 16 & \\
\hline CK14 & & & \\
\hline Positive & 45 & 1 & 0.0034 \\
\hline Negative & 71 & 18 & \\
\hline CD10 & & & \\
\hline Positive & 19 & 5 & 0.293 \\
\hline Negative & 97 & 14 & \\
\hline p63 & & & \\
\hline Positive & 15 & 2 & 0.769 \\
\hline Negative & 101 & 17 & \\
\hline
\end{tabular}

single use of maspin. Additionally, all 16 patients that were CK5/6-positive and EGFR-positive, with low histological differentiation and younger age were maspinpositive. Although the analyses of gene expression arrays is required to conclude that maspin is a marker of the basal-like subtype, our results suggested that maspin could be a candidate for a basal marker in $\mathrm{TN}$ breast cancer.

It has been hypothesized that maspin may regulate gene transcription in response to cellular stress induced by inflammation, tissue injury and remodeling [8], but its function attributable to an aggressive phenotype in some breast cancers remains to be resolved. We can consider three possible explanations. One is that basal differentiation could contribute to a more aggressive phenotype $[5,9]$. The second is a high intracellular 


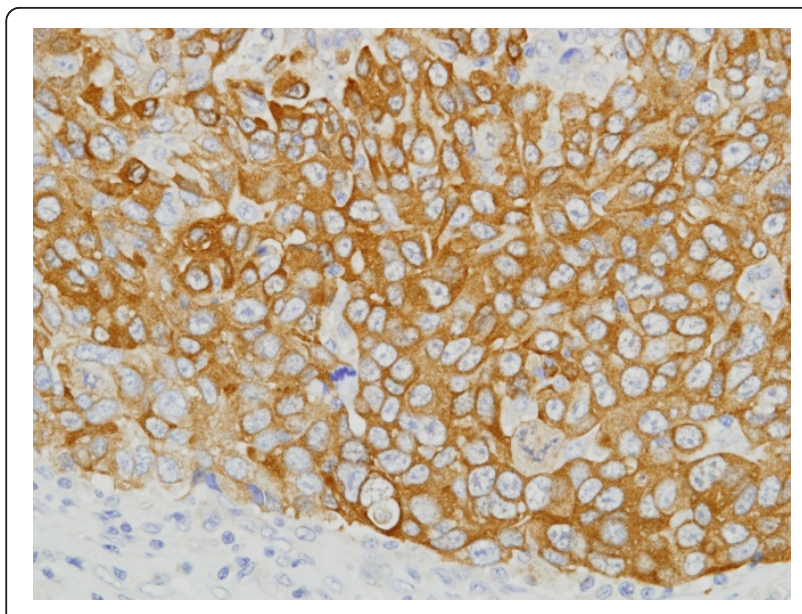

Figure 1 Immunohistochemical detection of maspin in triplenegative invasive ductal carcinoma. Cytoplasmic staining was noted in carcinoma cells, and stromal cells were negatively stained.

concentration of maspin resulting in auto-inhibition of its activity by non-covalent polymerization [10]. The third is mutation of the maspin gene causing loss of normal function of the maspin protein. Additionally, it has been reported that two intriguing genes upregulated by maspin re-expression were the E2F transcription factor 1 (E2F1) and the naturally occurring BRCA1 splice variant BRCA1b, and that maspin may play an important role in response to DNA damage at the level of cell-cycle regulation and cellular proliferation [8]. On the other hands, the majority of BRCA1-associated breast cancers is $\mathrm{TN}$ and expresses basal cytokeratins [11]. Therefore the elucidation of more precise molecular mechanisms between maspin and BRCA1 may be one of the important targets of future research. It could be argued that instead of identifying descriptive and prognostic molecular subgroups, such as basal-like within TN breast cancer, it would be more clinically relevant to identify patients whose TN tumors are sensitive to specific chemotherapy agents and targeted therapies [12]. It is important for the development of

Table 4 Association between maspin expression and basal-like subtype in triple-negative breast cancer

\begin{tabular}{|c|c|c|c|}
\hline & \multicolumn{2}{|c|}{ Maspin expression } & \multirow[b]{2}{*}{$\begin{array}{c}P- \\
\text { value }\end{array}$} \\
\hline & $\begin{array}{c}\text { Positive }(85.9 \%) \\
116 \text { cases }\end{array}$ & $\begin{array}{c}\text { Negative }(14.1 \%) \\
19 \text { cases }\end{array}$ & \\
\hline \multicolumn{4}{|c|}{ CK5/6 and/or EGFR } \\
\hline Positive & 92 & 11 & 0.041 \\
\hline Negative & 24 & 8 & \\
\hline \multicolumn{4}{|c|}{$\begin{array}{l}\text { CK5/6 and/or EGFR } \\
\text { and/or CK14 }\end{array}$} \\
\hline Positive & 96 & 11 & 0.013 \\
\hline Negative & 20 & 8 & \\
\hline
\end{tabular}

targeted therapies to elucidate the role and function of maspin in $\mathrm{TN}$ breast cancer.

\section{Acknowledgements}

We are grateful to Ms Shinobu Haraguchi and Ms Yoshie Jitouhou for their excellent technical assistance.

\section{Author details}

'Department of Molecular and Cellular Pathology, Field of Oncology, Kagoshima University Graduate School of Medical and Dental Sciences 8-351, Sakuragaoka, Kagoshima 890-8544, Japan. ${ }^{2}$ Hakuaikai Sagara Hospital, Kagoshima, 3-31, Matsubara, Kagoshima 892-0833, Japan.

\section{Authors' contributions}

YU designed the study, evaluated immunohistochemistry, performed statistical analysis and wrote the manuscript. YO interpreted the data and evaluated immunohistochemistry. MS performed immunohistochemical experiments. YR, YS, YS and ST participated in the sampling of clinical data. AT performed critical reading of manuscript and supervision. All authors have read and approved the final manuscript.

\section{Competing interests}

The authors declare that they have no competing interests.

Received: 6 January 2011 Accepted: 16 April 2011

Published: 16 April 2011

\section{References}

1. Zou Z, Anisowicz A, Hendrix MJ, Thor A, Neveau M, Sheng S, Rafidi K, Seftor E, Sagar R: Maspin, a serpin with tumor-suppressing activity in human mammary epithelial cells. Science 1994, 263:526-529.

2. Rakha EA, Ellis IO: Triple-negative/basal-like breast cancer: review. Pathology 2009, 41:40-47.

3. Umekita Y, Ohi Y, Sagara Y, Yoshida H: Expression of maspin predicts poor prognosis in breast cancer patients. Int J Cancer 2002, 100:452-455.

4. Umekita Y, Yoshida H: Expression of maspin is up-regulated during the progression of mammary ductal carcinoma. Histopathology 2003, 42:541-545.

5. Rakha EA, Putti TC, El-Rehim DM, Paish C, Green AR, Powe DG, Lee AH, Robertson JF, Ellis IO: Morphological and immunophenotypic analysis of breast carcinomas with basal and myoepithelial differentiation. J Pathol 2006, 208:495-506

6. Umekita Y, Souda M, Ohi Y, Sagara Y, Rai Y, Takahama T, Yoshida H: Expression of wild-type estrogen receptor $\beta$ protein in human breast cancer: Specific correlation with HER2/neu overexpression. Pathol Int 2006, 56:423-427.

7. Nielsen TO, Hsu FD, Jensen K, Cheang M, Karaca G, Hu Z, HernandezBoussard T, Livasy C, Cowan D, Dressler L, Akslen LA, Ragaz J, Gown Am, Gilks CB, van de Rijn M, Perou CM: Immunohistochemical and clinical characterization of the basal-like subtype of invasive breast carcinoma. Clin Cancer Res 2004, 10:5367-5374.

8. Bailey CM, Khalkhali-Ellis Z, Seftor EA, Hendrix MJC: Biological function of maspin. J Cell Phisiol 2006, 209:617-624.

9. Jones C, Nonni AV, Fuitord L, Merrett S, Chaggar R, Eusebi V, Lakhani SR: CGH analysis of ductal carcinoma of the breast with basaloid/ myoepithelial cell differentiation. Br J Cancer 2001, 85:422-427.

10. Liu T, Pemberton PA, Robertson AD: Three-state unfolding and self association of maspin, a tumor-suppressing serpin. J Biol Chem 1999, 274:29628-29632.

11. Rakha EA, Reis-Filho JS, Ellis IO: Basal-like breast cancer: A critical review. $J$ Clin Oncol 2008, 26:2568-2581.

12. Foulkes WD, Smith IE, Reis-Filho JS: Triple-nagtive breast cancer. New Engl J Med 2010, 363:1938-1948.

\section{doi:10.1186/1746-1596-6-36}

Cite this article as: Umekita et al:: Maspin expression is frequent and correlates with basal markers in triple-negative breast cancer. Diagnostic Pathology 2011 6:36. 